\title{
BMJ Open Microscopic changes in the spinal extensor musculature in patients experiencing chronic spinal pain: protocol for a systematic review
}

\author{
Shilpa Purushotham, ${ }^{1,2}$ Robert Stanley Stephenson, ${ }^{2}$ Andy Sanderson, ${ }^{1,3}$ \\ Deborah Falla (i) ${ }^{1}$
}

To cite: Purushotham S, Stephenson RS, Sanderson A, et al. Microscopic changes in the spinal extensor musculature in patients experiencing chronic spinal pain: protocol for a systematic review. BMJ Open 2021;11:e042729. doi:10.1136/ bmjopen-2020-042729

- Prepublication history and supplemental material is published online only. To view please visit the journal online (http://dx.doi.org/10.1136/ bmjopen-2020-042729).

Received 13 July 2020 Revised 14 January 2021 Accepted 09 February 2021

Check for updates

(C) Author(s) (or their employer(s)) 2021. Re-use permitted under CC BY-NC. No commercial re-use. See rights and permissions. Published by BMJ.

${ }^{1}$ Centre of Precision Rehabilitation for Spinal Pain (CPR Spine), School of Sports, Exercise and Rehabilitation Sciences, University of Birmingham, Birmingham, UK ${ }^{2}$ Anatomy Department, Birmingham Medical School, College of Medical and Dental Sciences, University of Birmingham, Birmingham, UK ${ }^{3}$ Department of Sport and Exercise Sciences, Musculoskeletal Science and Sports Medicine Research Centre, Manchester Metropolitan University, Manchester, UK

Correspondence to Professor Deborah Falla; d.falla@bham.ac.uk

\section{ABSTRACT}

Introduction Chronic spinal pain (CSP) is the most common musculoskeletal disorder and is a leading cause of disability as per the Global Burden of Diseases. Previous reviews of microscopic changes in the spinal extensor muscles of people with CSP have focused on the lumbar region only and the results have been inconclusive. Therefore, in this protocol, we aim to assess microscopic changes in the extensor muscles of all spinal regions, investigating regionally specific changes in muscle fibre types of the spinal extensor muscles in patients with nonspecific CSP.

Methods/analysis This protocol was designed using Preferred Reporting Items for Systematic Review and Meta-Analysis Protocols (PRISMA-P) guidelines. Searches will use the following databases: MEDLINE, Embase, PubMed, CINAHL Plus and Web of Science along with relevant grey literature searches. Two reviewers will conduct the searches, perform data extraction, apply inclusion criteria and conduct risk of bias assessment using Newcastle-Ottawa Scale. Data will be synthesised and analysed independently. If there is sufficient homogeneity, then meta-analysis will be conducted by the reviewers jointly. If not, meta-synthesis or narrative reporting will be performed. The quality of the evidence will be assessed using Grading of Recommendations, Assessment, Development and Evaluation (GRADE) guidelines.

Ethics and dissemination The results of this study will be submitted for publication to a peer-reviewed journal and will be presented at conferences. Ethical approval for this systematic review was not required due to no patient data being collated.

PROSPERO registration number CRD42020198087.

\section{INTRODUCTION}

Chronic spinal pain (CSP) is one of the most common musculoskeletal disorders with a lifetime prevalence of $54 \%$ to $80 \% .{ }^{1} \mathrm{CSP}$ is the leading cause of disability ${ }^{2}$ and apart from the impact on quality of life, it produces a massive socioeconomic burden. ${ }^{13-8}$ In most cases of CSP, no specific nociceptive source can be found and a diagnosis of non-specific chronic
Strengths and limitations of this study

- This is the first systematic review to investigate the microscopic changes in the spinal extensor musculature of the cervical, thoracic and lumbar regions in a chronic spinal pain population.

- This review allows for comparison of microscopic changes within and between different regions of the spine.

- This review will use the Newcastle-0ttawa Scale which is the most suitable tool to assess the risk of bias across various observational studies.

While it would also be valuable to consider the macroscopic changes in the spinal musculature, this review will be limited to microscopic changes only.

pain or persistent pain is provided. ${ }^{79}$ As per the National Institute for Health and Care Excellence (NICE) guidelines, chronic pain is defined as any long term pain or persistent pain that 'persists or recurs for longer than 3 months'. 1011

Low back pain (LBP) is the most common spinal pain complaint with a lifetime prevalence of $84 \%$. The second most common spinal pain complaint is neck pain, with up to $70 \%$ of people experiencing at least one episode of neck pain in their lifetime. ${ }^{12}$ In the case of thoracic spinal pain, though considered a common complaint and similarly disabling like lumbar and cervical spinal pain, the associated epidemiology is not well documented. ${ }^{13}$

Structural changes have been observed in the spinal musculature in people experiencing CSP compared with an asymptomatic population. These changes can occur on a macroscopic or microscopic level within the muscle. Macroscopic changes include fatty infiltration, reduced cross sectional area (CSA) or atrophy. Microscopic changes include remodelling of muscle fibre types, 
change in their size and distribution. ${ }^{14-17}$ These changes may lead to reduced function and have implications for ongoing pain and disability. ${ }^{18} 19$ A comprehensive understanding of the macroscopic and microscopic features of the skeletal muscles in the spinal region in people with CSP is needed to better understand the pathophysiology associated with chronic pain. ${ }^{18}$

Five systematic reviews have examined changes in macroscopic features of spinal musculature in people with CSP including changes in the CSA of muscles, fatty infiltration and muscle atrophy using various imaging techniques. ${ }^{20-24}$ Two of these reviews were specific to $\mathrm{LBP}^{2024}$ and one of these two also examined changes in microscopic features of spinal musculature. ${ }^{24}$ Ranger et al, reported conflicting evidence to support an association between the CSA of spinal muscles and LBP. ${ }^{20}$ They also reported that the CSA of multifidus had a negative association with LBP intensity and was predictive of the occurrence of LBP for up to 12 months in men. ${ }^{20}$ Goubert $e t$ al, reported that paraspinal muscular atrophy was present in people with chronic LBP, except for the erector spinae. ${ }^{24}$ There were also inconsistent results regarding fatty infiltration of lumbar muscles in LBP in both these studies ${ }^{20}{ }^{24}$ but both reviews consistently documented atrophy of multifidus in people with chronic LBP. Two of the five systematic reviews examined macroscopic changes of the neck musculature in people with neck pain. ${ }^{21} 22$ De Pauw et al reviewed morphological changes using ultrasonography and MRI in chronic non-specific neck pain as well as in whiplash associated disorders (WAD). This work revealed that the neck extensor muscles have decreased CSA in people with non-specific neck pain, yet increased CSA in WAD. ${ }^{21}$ They also found evidence for fatty infiltration in the extensor muscles in WAD and a cephalocaudal decline of fat in the suboccipital muscles in people with chronic neck pain, with the exception of the multifidus. ${ }^{21}{ }^{25}$ The most recent review by Farrell $\mathrm{et} \mathrm{al}$, assessing the musculature of the cervical spine in WAD and non-specific neck pain by MRI, could not draw any conclusions mostly due to low quality of evidence (owing to small sample sizes and greater heterogeneity). However, they reported a significantly decreased CSA of the rectus capitis posterior major muscle in people with chronic neck pain compared with controls. ${ }^{22}$

Overall, since these systematic reviews conducted on the macroscopic features of the paraspinal musculature are high quality reviews (exhibiting moderate to high confidence on AMSTAR 2 tool scoring ${ }^{26}$ ), we are confident in what these studies conclude regarding macroscopic changes in people with CSP.

In contrast, only two systematic reviews have studied microscopic features (eg, quantification of the proportional area of different fibre types within a muscle based on size and distribution) of the spinal musculature in people with CSP and both were focused on LBP only. ${ }^{19} 24$ The review by Cagnie et al yielded inconclusive results for fibre typing between different intrinsic lumbar muscles, however, some evidence in this review suggested that the
CSA occupied by type 1 fibres in women was relatively higher compared with men in both healthy and patients with LBP. ${ }^{19}$ It should be noted that this review included studies which were not limited to chronic non-specific LBP and the inclusion criteria did not consider the quality of the selected studies. Goubert et al concluded that there was no fibre type abnormalities in the paraspinal muscles of people with chronic LBP, but this review considered only case-control studies which is indicative of restricted evidence. ${ }^{24}$ No previous systematic review has examined microscopic changes in the spinal musculature in the cervical or thoracic region.

Thus, here we aim to systematically review the primary studies examining muscle fibre type distribution in people with CSP considering the cervical, thoracic and lumbar regions. Specifically, our objectives are to:

a. Assess muscle fibre type composition and density/proportion of muscle fibre types within the spinal extensor muscles in patients experiencing CSP compared with healthy controls.

b. Compare muscle fibre type composition and density/ proportion of muscle fibre types in the spinal extensor muscles across different regions of the spine in people with chronic neck, thoracic or low back pain.

\section{METHODS}

\section{Design}

This systematic review was designed using PRISMA-P guidelines (Preferred Reporting Items for Systematic Reviews and Meta-Analysis Protocols)(online supplemental file 1$)^{27}$ and in accordance with the Cochrane Handbook for Systematic Reviews. ${ }^{28}{ }^{29}$ This protocol is registered under PROSPERO, ID number CRD42020198087.

\section{Inclusion criteria}

In order to report the characteristics of the study, the adapted Population, Intervention, Comparison, Outcomes and Study (PICOS) framework ${ }^{30}$ was used to develop the eligibility criteria.

- Population: Studies including adult's $\geq 18$ years of age experiencing non-specific CSP. For this review purposes, spinal pain is considered chronic if persisting $>3$ months as per NICE guidelines. ${ }^{10}$ Spinal regions considered for this review include cervical, thoracic and lumbar regions.

- Comparator: Studies must compare the microscopic changes in the spinal extensor musculature (cervical, thoracic or lumbar region) and this comparison could be with healthy controls ( $\geq 18$ years) or between different extensor muscles of the spine or the same muscle in different regions of the spine.

- Outcome: The muscle fibre type composition and/or density/proportion of muscle fibre types in extensor muscles of the spine. The muscles of interest are the intrinsic muscles of the back, for example, erector spinae and multifidus. 
- Study design: Observational studies will likely constitute the highest level of evidence for this review, as ascertained by scoping searches.

\section{Exclusion criteria}

- Studies including specific spinal pain due to acute trauma, fractures, surgeries, deformities like scoliosis, kyphosis, lordosis or pathologies like inflammatory disorders, infection and malignancy, or other relevant pathologies.

- Studies comparing exercises/interventions while looking for microscopic changes in spinal muscles, as these studies are investigating the influence of exercise on muscle fibres in back pain rather than the influence of back pain on muscle fibres.

- Studies that are not in English language.

- Studies where the full text is not available.

\section{Information sources}

Electronic databases MEDLINE (Ovid Interface), Embase, PubMed, CINAHL Plus and Web of Science will be searched for relevant studies. Searches will be conducted from database inception until March 2021. Key journals including Journal of Anatomy, The Spine Journal, European Spine Journal and the Clinical Journal of Pain will be searched. Any anticipation of publication bias will be assessed by searching thoroughly for unpublished literature from conference proceedings from 2018 to 2020, including Society for Back Pain Research Annual Meeting and the World Institute of Pain. Grey literature searching will be conducted by searching the Open Grey and British National bibliography databases. Hand searching of reference lists from eligible studies will also be carried out. Study authors from the included studies will be contacted through email for any details if necessary.

\section{Search strategy}

Search strategies has been developed using free-text or MeSH (MedicalSubject Headings) terms applying PICOS criteria for specific databases. An example of a comprehensive search strategy developed for the MEDLINE (Ovid) database is detailed in box 1. The lead author (SP) will conduct the searches from all the information sources, identify all the potentially eligible studies and exclude duplicates.

\section{STUDY RECORDS}

\section{Data management}

The abstracts and citations of all potentially relevant studies obtained from the literature search will be imported into EndNote X9 (Clarivate Analytics) data management software. Full-texts of all potentially eligible studies after review of titles and abstracts will be imported into Endnote X9 for thorough screening. The search strategy was based on the eligibility criteria.
Box 1 Comprehensive search strategy developed for MEDLINE (Ovid) database.

1. exp Neck Pain/ or exp Back Pain/ or exp Chronic Pain/ or spinal pain.mp. or exp Low Back Pain/

2. exp Chronic Pain/ or exp Back Pain/ or chronic back pain.mp. or exp Low Back Pain/

3. exp Back Pain/ or exp Low Back Pain/ or persistent back pain.mp.

4. exp Neck Pain/ or exp Back Pain/ or cervical spinal pain.mp. or exp Low Back Pain/

5. neck pain.mp. or exp Neck Pain/

6. exp Chronic Pain/ or exp Neck Pain/ or chronic neck pain.mp.

7. exp Neck Pain/ or persistent neck pain.mp. or exp Chronic Pain/

8. exp Back Pain/ or exp Low Back Pain/ or thoracic spinal pain.mp.

9. Spinal Diseases/ or persistent thoracic spine pain.mp.

10. exp Low Back Pain/ or lumbar spinal pain.mp. or exp Chronic Pain/ or exp Back Pain/

11. lumbago.mp. or exp Low Back Pain/

12. exp Chronic Pain/ or exp Low Back Pain/ or exp Back Pain/ or low* back pain.mp.

13. exp Low Back Pain/ or exp Back Pain/ or chronic low* back pain. mp. or exp Chronic Pain/

14. exp Chronic Pain/ or exp Low Back Pain/ or persistent low* back pain.mp.

15. exp Low Back Pain/ or exp Back Pain/ or LBP.mp.

16. exp Low Back Pain/ or CLBP.mp. or exp Chronic Pain/

17. 1 or 2 or 3 or 4 or 5 or 6 or 7 or 8 or 9 or 10 or 11 or 12 or 13 or 14 or 15 or 16

18. exp Muscle Fibers, Slow-Twitch/ or exp Muscle Fibers, Fast-Twitch/ or exp Muscle Fibers, Skeletal/ or muscle fibre type.mp.

19. exp Muscle Fibers, Fast-Twitch/ or exp Muscle Fibers, Slow-Twitch/ or exp Muscle Fibers, Skeletal/ or muscle fiber type.mp. or exp Myosin Heavy Chains/

20. exp Muscle Fibers, Skeletal/ or fibre size.mp.

21. fiber size.mp. or exp Muscle Fibers, Skeletal/ or exp Muscular Atrophy/

22. fibre distribution.mp. or exp Muscle Fibers, Skeletal/

23. exp Muscle Fibers, Skeletal/ or fibre area.mp.

24. fiber characteristics.mp. or exp Myosin Heavy Chains/ or exp Muscle Fibers, Skeletal/

25. exp Muscle Fibers, Fast-Twitch/ or exp Muscle Fibers, Skeletal/ or exp Muscle Fibers, Slow-Twitch/ or fibre characteristics.mp.

26. exp Muscle Fibers, Skeletal/ or fibre proportion.mp.

27. muscle fibre density.mp. or exp Muscle Fibers, Skeletal/

28. exp Muscle Fibers, Slow-Twitch/ or exp Muscle Fibers, Fast-Twitch/ or exp Muscle Fibers, Skeletal/ or fibre type composition.mp.

29. exp Muscle Fibers, Skeletal/ or microscopy of back muscle*.mp.

30. 18 or 19 or 20 or 21 or 22 or 23 or 24 or 25 or 26 or 27 or 28 or 29

31. 17 and 30

32. limit 31 to humans

\section{Selection process}

Following the removal of duplicates, two reviewers (SP/ AS) will independently screen all the eligible studies to assess each studies' suitability for inclusion in this review. In the first stage, the title and abstract of all studies obtained from search results will be screened and ineligible studies will be excluded. All potentially eligible studies, and any studies where eligibility is unclear following title and abstract screening, will be 
Table 1 List of items that will be extracted from all the eligible studies

\begin{tabular}{ll}
\hline $\begin{array}{l}\text { Background } \\
\text { information }\end{array}$ & Study ID/author \\
\hline & Title of the study \\
& Year of publication \\
& Country of study \\
& Source of funding \\
& Aims/objectives of the study \\
\hline Methodology & Study design \\
& Sample size \\
\hline
\end{tabular}

Patient population characteristics - age, gender, diagnosis, confounding factors (like restriction of activity level)

Control population characteristics - age, gender, any confounding factors

Spinal pain characteristics - average duration of pain, laterality, severity

Biopsy samples - type of biopsy done and how muscle sample was obtained (eg, surgical, ultrasound guided,), from which muscle, landmark site/location taken from, unilateral or bilateral region

Synthesis of Analysis and outcome measures - fibre type results (I, Ila, Ilb/llc, IIx) slow, intermediate or fast twitch, fibre type percentage/ distribution, fibre size, area occupied by fibre types, any other fibre characteristics if mentioned

$\begin{array}{ll}\begin{array}{l}\text { Study } \\ \text { limitations }\end{array} & \begin{array}{l}\text { Confounding factors, observational bias, } \\ \text { measurement techniques }\end{array}\end{array}$

further screened for eligibility by reading the full text. Any disagreement between reviewers at each stage will be discussed between reviewers. If reviewers are unable to reach consensus, then the third reviewer (DF) will be consulted to make a decision.

\section{Data collection process}

Following the screening process, all relevant information will be extracted from each included study by the lead author (SP), following which accuracy will be checked by the second reviewer (AS). A standardised data extraction form has been developed based on the Cochrane data extraction template and guided by the objectives and inclusion criteria of the review. This form will be piloted on a small number of studies to confirm the completeness of data extraction.

\section{Data items}

Data extraction items of interest to this review are summarised in table 1, and includes: author (study ID), the title of the paper, year of publication, country of study, source of funding, aims/objectives of the study, study design, sample size, characteristics of patient and control population, spinal pain characteristics, details of biopsy samples, analysis and outcome measures from the studies, muscle fibre type, distribution, fibre size, proportional area of different fibre types, any other fibre characteristics confounding factors and study limitations. If any data is omitted or ambiguous from the studies or its results affecting its eligibility, then the respective author will be contacted via email for clarifications. If there is no response from the author within 2 weeks, a reminder email will be sent allowing a further 2 weeks for a response. If there is still no response then the study will not be considered for inclusion but it will be noted as excluded for ambiguity, with specific reason noted.

\section{Outcomes and prioritisation}

The primary outcome that will be considered in this review is the muscle fibre type distribution (Type 1 - slow twitch-oxidative, Type II a - fast twitch-oxidative glycolytic, Type II b (II x) - fast twitch-glycolytic, Type II c - intermediate) and the proportional area of different fibre types determined by the fibre size and distribution in spinal extensor muscles of patients with CSP. We will also consider additional outcomes such as gender differences within these results (if there are sufficient primary studies addressing this).

\section{Risk of bias in individual studies}

Risk of Bias (RoB) will be assessed by the two reviewers independently using the Newcastle-Ottawa Scale (NOS). Since this review anticipates observational studies, the NOS is the most suitable tool as this is developed to assess the quality of observational studies. ${ }^{31}$ When we identify the study designs used, we will ensure that the version of the NOS used matches the design of the evidence (eg, using the case-control or cohort versions). The NOS considers three main categories for the scoring system which include selection, comparability and outcome/ exposure. Under selection, a study can get maximum of 4 stars, maximum of 2 stars for comparability and maximum 3 stars for outcome. ${ }^{31}$ There are thresholds for converting NOS to the Agency for Healthcare Research and Quality standards. ${ }^{31}$ According to these, a study can be graded as (1) Good quality: if selection category scores 3 or 4 stars AND comparability scores 1 or 2 stars AND outcome scores 2 or 3 stars; (2) Fair quality: selection scores 2 stars AND comparability scores 1 or 2 stars AND outcome scores 2 or 3 stars; (3) Poor quality: selection scores 0 or 1 star OR comparability scores 0 stars OR outcome scores 0 or 1 stars.

Though previous studies have raised questions on its reliability and unknown validity, ${ }^{32}{ }^{33}$ there is growing use of NOS because of its ease and suitability in systematic reviews. ${ }^{33}$ Therefore, for this review, the NOS will be used as it is widely used and designed for use across observational studies.

\section{Data synthesis}

The data synthesis in this review will be influenced by the methodology and the results of all the studies included. If there is homogeneity in the methodology and outcome 
measures of studies, then meta-analysis will be considered. However, this decision can be made only following the data extraction. The meta-analysis will be completed if there is sufficient homogeneity in the following factors:

- Measurement technique for muscle fibre type measured as a percentage of distribution or as the proportional area occupied by each fibre type or by fibre size.

- Which muscles were studied? - Biopsies/muscle samples taken from different muscles of the same region or same muscle from different region or different muscles from different regions.

- Which population was considered?

- Age group- young adults, middle age or older adults.

- Pain-related factors, if mentioned in studies, such as pain intensity, activity/disability level which may influence the muscle morphology.

Where sufficient homogeneity is reached in the points above, meta-analysis will be conducted considering the following outcomes:

- Comparing a common histological outcome in the same extensor muscles across different regions of the spine (eg, multifidus, erector spinae).

- Comparing a common histological outcome in different extensor muscles of the same spinal region.

Reviewers will independently assess the homogeneity of the included studies and group/subgroup them appropriately for analysis of outcome measures. Further clarity on whether there are enough studies to analyse the areas of interest, and how best studies can be grouped/ subgrouped appropriately for precise comparisons, will be determined following data extraction. For metaanalysis, first the reviewers will independently determine the grouping, justifying where precise comparisons can be made based on outcome measures. If there is lack of clarity on the homogeneity of a study, inclusion will be decided by joint consensus. Assessment for subgroup analysis will also be considered in a similar manner (eg, comparison between men and women with LBP). If there are discrepancies between the two reviewers on inclusion of certain studies in the meta-analysis or subgroup analysis, or whether or not to conduct the meta-analysis at all, then a third reviewer (DF) will determine the potential for meta-analysis. ${ }^{34}$

If reviewers decide it may be suitable to carry out the meta-analysis, a statistical test for heterogeneity will be conducted to calculate the $\mathrm{I}^{2}$ value which will determine the total variation across the studies as a percentage. ${ }^{35} \mathrm{I}^{2}$ value can be around $25 \%, 50 \%$ or $75 \%$ indicating low, moderate or high heterogeneity between studies, respectively. ${ }^{2935}$ Similar to previous reviews, we will proceed with meta-analysis if the $\mathrm{I}^{2}$ value is $<50 \% .{ }^{34} 36$

If meta-analysis is determined to be pertinent, the outcome results from all the eligible studies will be collected in a systematic way. As we are investigating a binary outcome, that is, muscle fibre type changes present or absent in CSP, ORs will likely be the most suitable statistical approach. ${ }^{37}$ ORs will be used to investigate the relationship between muscle fibre types and CSP, clarifying the existence of muscle fibre type changes in CSP compared with healthy controls.

If the reviewers agree not to conduct meta-analysis due to the heterogeneity of pooled data, then metasynthesis will be considered under expert guidance. ${ }^{34}$ If the eligible studies cannot be pooled to conduct metasynthesis, we would then consider presenting our findings in a narrative way. In narrative reporting, there will be full interpretation of the findings for each outcome measure. The clinical and statistical significance of each outcome measure, as well as the RoB assessments of individual studies will be considered. The findings will be summarised for all outcomes of interest and inferences drawn from the overall direction of effect.

\section{Confidence in cumulative evidence}

The Grading of Recommendations Assessment, Development and Evaluation (GRADE) approach will be applied to assess the quality of evidence. ${ }^{38}$ GRADE tool will be carried out as per the guidelines for our outcome measures identified previously. The GRADE approach determines the overall cumulative quality of the evidence across individual studies based on five determinants - (1) risk of bias, ${ }^{39}$ (2) inconsistency - heterogeneity in studies in the review (eg, population, age group, chronicity and severity of spinal pain), ${ }^{40}$ (3) indirectness - for example, the use of results from cadaveric samples in some studies, (4) imprecision - with larger the sample sizes indicating greater precision ${ }^{41}$ and finally (5) publication bias arising from non-publication of relevant results. ${ }^{42}$ Since it is anticipated that most of the included studies will be observational studies with case-control, cohort and crosssectional designs, these studies are given a 'low' rating initially in the GRADE framework. The study can be downgraded or upgraded depending on the above determinants and subsequently, quality of evidence will be graded as either very low $(+)$, low $(++)$, moderate $(+++)$ or high $(++++)$ according to the GRADE guidelines. ${ }^{38}$ Quality of evidence can also be upgraded based on the large effect sizes or a large number of studies indicating the same direction of effect for the muscle fibre type distribution or the area occupied by the fibre types.

\section{Patient and public involvement}

The research question in this study forms part of a larger discussion within our patient and public involvement meetings. Patients will not be involved in the analysis and data collection of the systematic review.

\section{Discussion of implications of this study}

Studies have shown that pain can affect muscle behaviour which has the potential to induce histological/structural changes in the muscle fibres. ${ }^{43}$ These changes include alterations in the muscle fibre size and distribution and potentially transformation of fibre types. ${ }^{44}$ Some studies have suggested that changes in the size and distribution of different muscle fibre types are associated with the duration of pain ${ }^{14} 1745$ whereas others did not find any association. ${ }^{46}$ Some studies 
found significant differences in proportional area occupied by different fibre types ${ }^{46}$ and some did not. ${ }^{17}$ This systematic review aims to provide a comprehensive understanding of our current knowledge of microscopic changes in the spinal musculature in people with CSP. This knowledge can help to direct exercise prescription for the management of CSP with the aim of improving muscle function and potentially reverting 'pathological' or 'adverse' changes in muscle fibre type.

\section{Ethics and dissemination}

No research ethics is required for this systematic review due to no patient data being collated. Results of this review will be submitted to be published in a peer-review journal and presented at conferences.

\section{Twitter Deborah Falla @Deb_Falla}

Contributors All authors formulated the focus of the systematic review. SP is a $\mathrm{PhD}$ student and DF (lead supervisor) and RSS are supervisors. SP drafted the initial version of the protocol manuscript with DF, RSS and AS all providing guidance on topic, methodology and analyses. All authors reviewed and commented on each draft of the protocol. All authors have approved the final manuscript.

Funding The authors have not declared a specific grant for this research from any funding agency in the public, commercial or not-for-profit sectors.

Competing interests None declared.

Patient consent for publication Not required.

Provenance and peer review Not commissioned; externally peer reviewed.

Supplemental material This content has been supplied by the author(s). It has not been vetted by BMJ Publishing Group Limited (BMJ) and may not have been peer-reviewed. Any opinions or recommendations discussed are solely those of the author(s) and are not endorsed by BMJ. BMJ disclaims all liability and responsibility arising from any reliance placed on the content. Where the content includes any translated material, BMJ does not warrant the accuracy and reliability of the translations (including but not limited to local regulations, clinical guidelines, terminology, drug names and drug dosages), and is not responsible for any error and/or omissions arising from translation and adaptation or otherwise.

Open access This is an open access article distributed in accordance with the Creative Commons Attribution Non Commercial (CC BY-NC 4.0) license, which permits others to distribute, remix, adapt, build upon this work non-commercially, and license their derivative works on different terms, provided the original work is properly cited, appropriate credit is given, any changes made indicated, and the use is non-commercial. See: http://creativecommons.org/licenses/by-nc/4.0/.

\section{ORCID iD}

Deborah Falla http://orcid.org/0000-0003-1689-6190

\section{REFERENCES}

1 Manchikanti L, Singh V, Datta S, et al. Comprehensive review of epidemiology, scope, and impact of spinal pain. Pain Physician 2009;12:E35-70.

2 Briggs AM, Woolf AD, Dreinhöfer K, et al. Reducing the global burden of musculoskeletal conditions. Bull World Health Organ 2018;96:366-8.

3 Costa-Black KM, Loisel P, Anema JR, et al. Back pain and work. Best Pract Res Clin Rheumatol 2010;24:227-40.

4 Watson PJ, Main CJ, Waddell G, et al. Medically certified work loss, recurrence and costs of wage compensation for back pain: a follow-up study of the working population of Jersey. Br J Rheumatol 1998;37:82-6.

5 Hoy D, March L, Brooks P, et al. Measuring the global burden of low back pain. Best Pract Res Clin Rheumatol 2010;24:155-65.

6 Hoy DG, Protani M, De R, et al. The epidemiology of neck pain. Best Pract Res Clin Rheumatol 2010;24:783-92.

7 Hartvigsen J, Hancock MJ, Kongsted A, et al. What low back pain is and why we need to pay attention. Lancet 2018;391:2356-67.

8 James SL, Abate D, Abate $\mathrm{KH}$, et al. Global, regional, and national incidence, prevalence, and years lived with disability for 354 diseases and injuries for 195 countries and territories, 1990-2017: a systematic analysis for the global burden of disease study 2017 . The Lancet 2018;392:1789-858.

9 Foster NE, Anema JR, Cherkin D, et al. Prevention and treatment of low back pain: evidence, challenges, and promising directions. Lancet 2018;391:2368-83.

10 NICE guidelines. National Institute for health and care excellence guideline scope chronic pain: assessment and management 2018.

11 Bernstein IA, Malik Q, Carville S, et al. Low back pain and sciatica: summary of NICE guidance. BMJ 2017;356:i6748.

12 Fejer R, Kyvik KO, Hartvigsen J. The prevalence of neck pain in the world population: a systematic critical review of the literature. Eur Spine J 2006;15:834-48.

13 Briggs AM, Smith AJ, Straker LM, et al. Thoracic spine pain in the general population: prevalence, incidence and associated factors in children, adolescents and adults. A systematic review. BMC Musculoskelet Disord 2009;10:77.

14 Mannion AF, Käser L, Weber E, et al. Influence of age and duration of symptoms on fibre type distribution and size of the back muscles in chronic low back pain patients. Eur Spine J 2000;9:273-81.

15 Danneels LA, Vanderstraeten GG, Cambier DC, et al. Ct imaging of trunk muscles in chronic low back pain patients and healthy control subjects. Eur Spine J 2000;9:266-72.

16 Hodges PW, Danneels L. Changes in structure and function of the back muscles in low back pain: different time points, observations, and mechanisms. J Orthop Sports Phys Ther 2019;49:464-76.

17 Mannion AF, Weber BR, Dvorak J, et al. Fibre type characteristics of the lumbar paraspinal muscles in normal healthy subjects and in patients with low back pain. J Orthop Res 1997;15:881-7.

18 Elliott J, Sterling M, Noteboom JT, et al. The clinical presentation of chronic whiplash and the relationship to findings of MRI fatty infiltrates in the cervical extensor musculature: a preliminary investigation. Eur Spine J 2009;18:1371-8.

19 Cagnie B, Dhooge F, Schumacher C, et al. Fiber typing of the erector spinae and multifidus muscles in healthy controls and back pain patients: a systematic literature review. J Manipulative Physiol Ther 2015;38:653-63.

20 Ranger TA, Cicuttini FM, Jensen TS, et al. Are the size and composition of the paraspinal muscles associated with low back pain? A systematic review. Spine J 2017;17:1729-48.

21 De Pauw R, Coppieters I, Kregel J, et al. Does muscle morphology change in chronic neck pain patients? - A systematic review. Man Ther 2016;22:42-9.

22 Farrell SF, Smith AD, Hancock MJ, et al. Cervical spine findings on $\mathrm{MRI}$ in people with neck pain compared with pain-free controls: a systematic review and meta-analysis. J Magn Reson Imaging 2019;49:1638-54.

23 Owers DS, Perriman DM, Smith PN, et al. Evidence for cervical muscle morphometric changes on magnetic resonance images after whiplash: a systematic review and meta-analysis. Injury 2018;49:165-76.

24 Goubert D, Oosterwijck JV, Meeus M, et al. Structural changes of lumbar muscles in non-specific low back pain: a systematic review. Pain Physician 2016;19:E985-99.

25 Elliott J, Sterling M, Noteboom JT, et al. Fatty infiltrate in the cervical extensor muscles is not a feature of chronic, insidious-onset neck pain. Clin Radiol 2008;63:681-7.

26 Shea BJ, Reeves BC, Wells G, et al. Hamel C, Moran J 2017.

27 Shamseer L, Moher D, Clarke M, et al. Preferred reporting items for systematic review and meta-analysis protocols (PRISMA-P) 2015: elaboration and explanation. BMJ 2015;349:g7647.

28 Higgins JP, Li T, Deeks JJ. Choosing effect measures and computing estimates of effect. Cochrane Handbook for Systematic Reviews of Interventions 2019:143-76.

29 Higgins J, Green S. Cochrane handbook for systematic reviews of interventions [electronic resource] / edited by Julian Higgins and Sally Green 2008.

30 Methley AM, Campbell S, Chew-Graham C, et al. PICO, PICOS and spider: a comparison study of specificity and sensitivity in three search tools for qualitative systematic reviews. BMC Health Serv Res 2014; $14: 579$

31 Wells. The Newcastle-Ottawa scale (NOS) for assessing the quality of nonrandomized studies in MetaAnalysis, 2015.

32 Hartling L, Milne A, Hamm MP, et al. Testing the Newcastle Ottawa scale showed low reliability between individual reviewers. J Clin Epidemiol 2013;66:982-93.

33 Stang A. Critical evaluation of the Newcastle-Ottawa scale for the assessment of the quality of nonrandomized studies in metaanalyses. Eur J Epidemiol 2010;25:603-5.

34 Sanderson A, Rushton AB, Martinez Valdes E, et al. The effect of chronic, non-specific low back pain on superficial lumbar muscle 
activity: a protocol for a systematic review and meta-analysis. BMJ Open 2019;9:e029850.

35 Higgins JPT, Thompson SG, Deeks JJ, et al. Measuring inconsistency in meta-analyses. BMJ 2003;327:557-60.

36 Heneghan NR, Smith R, Tyros I, et al. Thoracic dysfunction in whiplash associated disorders: a systematic review. PLoS One 2018;13:e0194235.

37 Egger M, Smith GD, Phillips AN. Meta-Analysis: principles and procedures. BMJ 1997;315:1533-7.

38 Balshem H, Helfand M, Schünemann HJ, et al. Grade guidelines: 3 . rating the quality of evidence. $J$ Clin Epidemiol 2011;64:401-6.

39 Guyatt GH, Oxman AD, Vist G, et al. GRADE guidelines: 4. Rating the quality of evidence--study limitations (risk of bias). J Clin Epidemiol 2011;64:407-15.

40 Guyatt GH, Oxman AD, Kunz R, et al. GRADE guidelines: 7. Rating the quality of evidence--inconsistency. J Clin Epidemiol 2011;64:1294-302.
41 Guyatt GH, Oxman AD, Kunz R, et al. GRADE guidelines 6. Rating the quality of evidence--imprecision. J Clin Epidemiol 2011;64:1283-93.

42 Guyatt GH, Oxman AD, Montori V, et al. GRADE guidelines: 5 . Rating the quality of evidence--publication bias. J Clin Epidemiol 2011;64:1277-82.

43 Falla D, Farina D. Neuromuscular adaptation in experimental and clinical neck pain. J Electromyogr Kinesiol 2008;18:255-61.

44 Schomacher J, Falla D. Function and structure of the deep cervical extensor muscles in patients with neck pain 2013.

45 Mazis N, Papachristou DJ, Zouboulis P, et al. The effect of different physical activity levels on muscle fiber size and type distribution of lumbar multifidus. A biopsy study on low back pain patient groups and healthy control subjects. Eur J Phys Rehabil Med 2009;45:459-67.

46 Crossman K, Mahon M, Watson PJ, et al. Chronic low back painassociated paraspinal muscle dysfunction is not the result of a constitutionally determined "adverse" fiber-type composition. Spine 2004;29:628-34. 